\title{
Comparison of the airtraq laryngoscope to the direct laryngoscopy in the pediatric airway
}

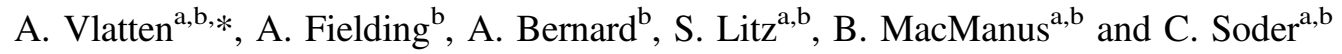 \\ ${ }^{\mathrm{a}}$ Departments of Pediatric Anesthesia and Pediatric Critical Care, IWK Health Centre, Halifax, NS, Canada \\ ${ }^{\mathrm{b}}$ Department of Anesthesia, Dalhousie University, Halifax, NS, Canada
}

Received 14 October 2010

Revised 2 December 2010

Accepted 3 January 2011

\begin{abstract}
Direct laryngoscopy (DL) is the most commonly used technique for tracheal intubation, but there is ongoing interest in new devices that have high success rates and are easily learned. The pediatric Airtraq (AT) is a recently developed intubation device that allows visualization of the glottis and intubation of the trachea without alignment of the oral, pharyngeal and tracheal axes. We studied the efficacy of the AT compared to the DL for laryngoscopy of young children with normal airway anatomy. In this prospective study, 49 children ( $5 \mathrm{yr}$ and younger) scheduled for elective surgery under general anesthesia were randomized into two groups: intubation using direct laryngoscopy (DL group) and laryngoscopy using the Airtraq (AT group). Time to best view, time to intubate, first attempt success rate (FASR), and percentage of glottic opening seen (percentage of glottis opening score) were recorded. Data are presented as median and interquartile range. Time to best view was five $(4,7)$ sec in DL and five $(4,7.5) \mathrm{sec}$ in AT. Time to intubate was $18(14.7,21) \mathrm{sec}$ in DL and $22.5(19.5,25.5) \mathrm{sec}$ in AT $(P=0.002)$. FASR was $100 \%$ in the DL and $83 \%$ in the AT. The percentage of glottis opening score was $80 \%$ (range $60-100 \%$ ) in the DL and $100 \%$ (range $100-100 \%)$ in the AT $(P<0.001)$. In young children with normal airway anatomy, the AT provides a better view of the glottis than the standard laryngoscope, but it takes longer to intubate the trachea and the FASR is lower.
\end{abstract}

Keywords: Airway, pediatric, video laryngoscope

\section{Introduction}

Direct laryngoscopy (DL) requires mechanical displacement of anatomic structures including the lips, jaw and tongue to create a direct line of sight to the laryngeal inlet. Even in expert hands, DL is not ideal for visualization of the larynx in children with anatomic anomalies such as Pierre Robin syndrome (Robin sequence). Poor visualization of the larynx may result in a failed intubation and may contribute to a failed airway. The failed airway remains a significant cause of morbidity and even mortality during emergency

*Corresponding author: Arnim Vlatten, Department of Pediatric Anesthesia and Department of Pediatric Critical Care, IWK Health Centre, 5850/5980 University Avenue, PO Box 9700 Halifax, B3K 6R8, Nova Scotia, Canada. Tel.: +1 902470 8251; Fax: +1 902470 7911; E-mail: arnim.vlatten@iwk.nshealth.ca. resuscitation and anesthesia [1]. It is not surprising that new intubation devices that promise better glottic visualization and easier tracheal intubation have generated considerable interest. One of the "look around the corner" devices, the Airtraq (AT) optical laryngoscope ${ }^{\mathrm{TM}}$ (Prodol, Vizcaya, Spain) uses a series of lenses and prisms to create an image of the larynx in a proximal viewfinder. The AT, recently introduced in pediatric and neonatal sizes, is a periscope-like device that contains two aligned channels in an anatomically shaped plastic housing: one channel contains the light source and optical components connected to an eyepiece while the second channel acts as a holder and conduit for the endotracheal tube. Adult manikin studies simulating normal and difficult airway anatomy have suggested that the AT provides superior intubating conditions and has a shorter 
learning curve compared to the DL [2,3]. Adult human studies have reached similar conclusions [4-7]. The AT has been used successfully in emergency cesarean section cases complicated by morbid obesity [8] and in cases where intubation by conventional means has failed [9]. No pediatric series has been published to date but case reports describe successful use of the AT optical laryngoscope in children with Treacher-Collins syndrome [10,11].

Since this device, designed for the difficult airway, has not been tested yet in the pediatric population, we conducted a prospective randomized trial comparing the AT optical laryngoscope to a standard laryngoscope used by experienced pediatric anesthesiologists for tracheal intubation in young children with normal airway anatomy. Studies on learning curves for new airway devices in students or novice anesthesiologists are needed as well as studies on the use of this device in the difficult pediatric airway. However, it is unethical to test a new airway device in a difficult scenario or by inexperienced practitioners without the proof that the new device is equal or superior in use by experienced practitioners to the current standard in the normal pediatric airway.

\section{Materials and methods}

Following institutional Research Ethics Board approval, 50 children (age five and under) scheduled for elective surgical procedures with the requirement of endotracheal intubation were enrolled. Informed parental consent was obtained. Exclusion criteria included previously documented difficult airway; predicted difficult bag mask ventilation; predicted difficult intubation; and need for rapid sequence induction.

The children were randomly assigned using a computer generated random number table to a DL group or an AT group. Children in the DL group were intubated using a Wisconsin one straight blade or a standard MacIntosh 2 curved blade based on the attending anesthesiologists' preferences. The choice was offered to compare AT laryngoscopy and intubation to the anesthesiologists' best normal technique. All of the anesthesiologists in this study perform DL by placing the tip of the blade in the valleculae. Children in the AT group were intubated using an infant AT (for tube size 2.5 to 4.0 ) or a pediatric AT (for tube size 4.5 to 5.5). Each intubation in the study was performed by one of five experienced pediatric anesthesiologists. Prior to participating in the study, each anesthesiologist viewed a training video produced by the AT manufacturer and performed three intubations on a pediatric manikin and five intubations on anesthetized children age 5 and under using the AT.

Induction of anesthesia was performed by inhalation of $8 \%$ sevoflurane in $60 \%$ nitrous oxide and $40 \%$ oxygen followed by intravenous injection of $2 \mathrm{mg} / \mathrm{kg}$ propofol prior to intubation. All children were monitored with electrocardiogram, non-invasive arterial blood pressure, pulse oximetry, capnography, and inhaled agent spectrophotometry. Before laryngoscopy, the lungs were pre-oxygenated with $100 \%$ oxygen for approximately $1 \mathrm{~min}$ using bag-mask ventilation. Cessation of spontaneous ventilation and absence of hemodynamic response to jaw thrust were used to determine that depth of anesthesia was adequate for intubation. None of the children received muscle relaxants prior to intubation and all intubations were performed with an uncuffed tracheal tube of appropriate size for age.

Time to intubation (TTI) was defined as the time interval between blade entry past the lips and the appearance of $\mathrm{CO}_{2}$ on the end-tidal tracing. TTI longer than $60 \mathrm{sec}$ was defined as a failed first attempt intubation. Time to best view (TTBV) was defined as the time interval between blade entry past the lips and the laryngoscopist's verbal declaration that the best view for endotracheal intubation was achieved. Timing was performed by a member of the research team using a stopwatch. Laryngeal best view was quantified by visually estimated percentage of glottis opening (POGO) score. First attempt success rate; use of external laryngeal manipulation performed by the intubator; complications associated with laryngoscopy-intubation; and loss of visualization due to "fogging" or "red-out" were recorded. Definition of "first attempt success" was correct placement of the tracheal tube within $60 \mathrm{sec}$ without oxygen saturation below $94 \%$ during the attempt. In the case of a failed first attempt, TTI was not assigned a value and was not included in the analysis. In general, the anesthesiologists were encouraged to get an optimal view to the vocal cords.

Parametric data were analyzed using a t-test and nonparametric data were analyzed using the MannWhitney rank sum test. Sample size was based on comparable adult studies. A $P<0.05$ was deemed clinically significant. The primary outcome variable in this study was the time to intubate for both devices. 


\section{Results}

Of the 50 children recruited, one patient in the AT group was removed from the study prior to intubation because of an unanticipated last-minute change in the airway management plan.

Patient characteristics of the 25 children in the DL group and the 24 children in the AT group are shown in table 1 . There was no significant difference in TTBV between the AT and DL groups $(P=0.418)$ (Table 2$)$. TTI was significantly longer in the AT group than in the DL group $(P=0.002)$ (Table 2). POGO scores were significantly higher in the AT group than in the DL group $(P<0.001)$ (Table 2, Fig. 1$)$. There was significantly less use of external laryngeal manipulation in the AT group (0/24) than in the DL group (12/25) (Chi-square $P<0.01)$. There were four failed intubations in the AT group and zero failed intubations in the DL group. Complications related to intubation, visual "red-out" or "fogging", and patient oxygen saturations below $94 \%$ were not seen in either group.

\section{Discussion}

This is the first clinical trial of the AT optical laryngoscope for intubation of the pediatric airway. Previous pediatric studies of other alternate airway devices including the STORZ Videolaryngoscope ${ }^{\mathrm{TM}}$ (Karl Storz, Tuttlingen, Germany), Glidescope ${ }^{\mathrm{TM}}$ (Verathon,

\section{Table 1}

Patient characteristics. Data present median, interquartile range and $\min -\max$

\begin{tabular}{lcc}
\hline Features & Airtraq $(\mathrm{n}=24)$ & $\begin{array}{c}\text { Direct laryngoscopy } \\
(\mathrm{n}=25)\end{array}$ \\
\hline Age $(\mathrm{mo})$ & $39(17.5,54.5[5-71])$ & $40(21.5,58[5-71])$ \\
Weight $(\mathrm{kg})$ & $14.3(10.3,16.7$ & $14.6(10.8,17.8$ \\
& $[7.5-24.5])$ & $[5.4-14.6])$ \\
Gender (male/ & $18 / 6$ & $17 / 8$ \\
female) & & \\
\hline
\end{tabular}

Bothell, WA, USA), and TrueView EVO2 laryngoscope $^{\mathrm{TM}}$ (Truphatek International Ltd, Netanya, Israel) have shown improved visualization of the larynx but longer intubation times [12-16]. Our study demonstrates similar findings: the AT provides a $20 \%$ better view of the pediatric larynx than the standard laryngoscope but increases the time required to achieve tracheal intubation by $25 \%$. Intubating with the AT took in $50 \%$ of the cases $4.5 \mathrm{sec}$ longer, statistically significant but with questionable clinical significance. However, the significantly higher POGO scores in the AT group support the claim that the AT provides excellent visualization of the pediatric larynx. Also encouraging is our finding that the AT device was not affected by "fogging" or "red-out", visual problems that often confound other indirect visualization techniques.

Despite the excellent laryngeal views observed in the AT group, the anesthesiologists experienced some difficulties with placement of the tracheal tube. Since TTBV was the same in both groups, we conclude that the prolonged time to intubate with the AT must have been caused by difficulties experienced after optimal visualization was achieved. It is possible that the difficulties

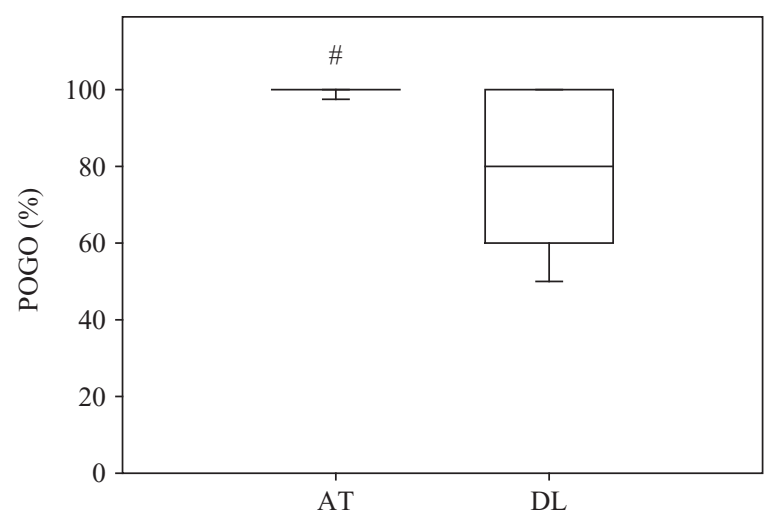

Fig. 1. Comparison of percentage of glottis opening seen between Airtraq AT and direct laryngoscopy. Data are median, interquartile range and 5-95\% interquartile range. \# AT versus direct laryngoscopy $(P<0.05)$.

Table 2

Comparison of time to best view, time to intubate and percentage of glottis opening for Airtraq and direct laryngoscope. Data are median, interquartile range and min-max

\begin{tabular}{lcc}
\hline Features & Airtraq & Direct laryngoscope \\
\hline Time to best view $(\mathrm{sec})$ & $5(4,7.5[3-28]) \mathrm{n}=24$ & $5(4,7[3-10]) \mathrm{n}=25$ \\
Time to intubate $(\mathrm{sec})$ & $22.5(19.5,25.5[14-50]) \mathrm{n}=20^{*}$ & $18(14.7,21[6-26]) \mathrm{n}=25$ \\
Percentage of glottis opening $(\%)$ & $100(100,100[90-100]) \mathrm{n}=24^{*}$ & $80(60,100[40-100]) \mathrm{n}=25$ \\
\hline
\end{tabular}

*Airtraq versus direct laryngoscope $(P<0.05)$. 
experienced reflect the fact that the anesthesiologist in this study were experienced laryngoscopists but inexperienced with the AT device. There are features inherent to the AT that requires a significant paradigm shift for the experienced anesthesiologist. The AT employs a fixed channel to guide the tracheal tube into the larynx. Direct manipulation of the tube is thus limited to forward and backward movement-angulation can only be modified by manipulating the AT device. This is initially frustrating for the expert direct laryngoscopist who is trained to precisely manipulate a tracheal tube in three dimensions. The anesthesiologists noted additional complexities unique to pediatric use of the AT device. In adult patients, the optical image of the larynx occupies most of the field of view provided by the eyepiece. In small children, the fully visualized larynx occupies a much smaller portion of the field of view. Commonly, on initial view, the larynx occupies the anterior quadrant of the AT eye piece. Advancing the tracheal tube in this position causes the tube to pass below the larynx into the esophagus $[11,17]$. To prevent this, the AT device must be pulled anteriorly until the centre of the larynx appears at the center of the eyepiece $[11,17,18]$. A second, more subtle observation may further explain the difficulties encountered: the AT optical path points $15^{\circ}$ to the right of the central axis of the instrument while the intubating channel points $15^{\circ}$ to the left. While this appears to be ideal for adult intubations, in children the tip of the scope is closer to the larynx and the offset of the two axes creates a problem. When the device is positioned to provide an ideal axial view of the larynx (Fig. 2a), the intubation channel axis is oriented towards the left ary-epiglottic fold and the advancing tracheal tube tends to become trapped in the vestibule or may miss the larynx completely and advance into the piriform fossa (Fig. 2b). To provide an optimal angle for intubation the operator must rotate the AT $15^{\circ}$ to the right (clockwise), providing an off-axis view of the larynx centered on the right vocal cord (Fig. 3a), but aligning the intubation channel axis with that of the trachea (Fig. 3b).

The infant AT is designed to accommodate tube sizes 2.5 to 4.0 and the pediatric AT is designed to accommodate endotracheal tubes size 4.5 to 5.5 . Two failed AT intubations occurred with the use of tubes at the upper size range of the pediatric device: a 5.5 tube in one case and a 5.0 tube in the other case. In both cases, despite good lubrication of the tubes, the anesthesiologist experienced considerable resistance to the passage of the tube down the intubation channel and the AT intubation attempt was abandoned after a TTI of $60 \mathrm{sec}$. The same tube was easily placed

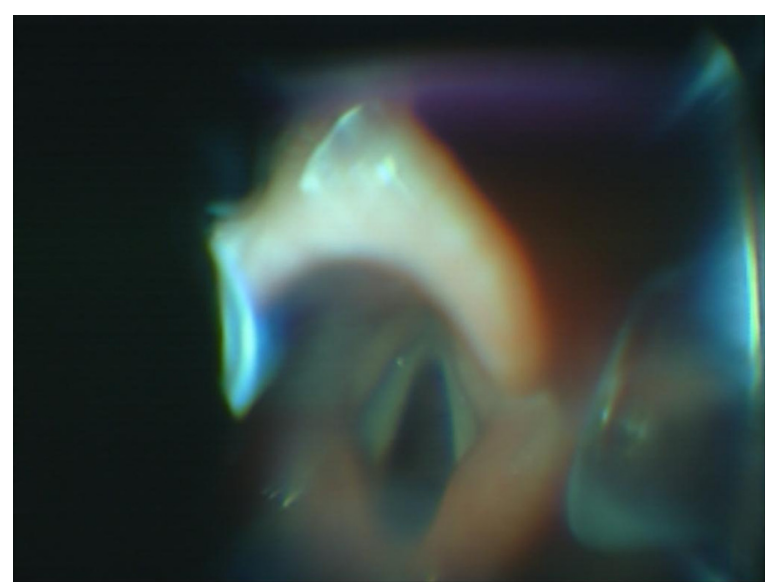

(a)

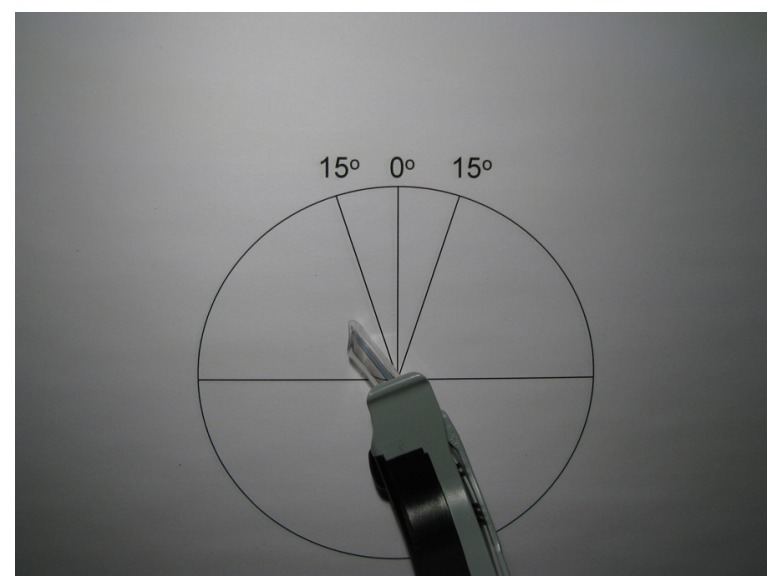

(b)

Figs $2 \mathrm{a}$ and $2 \mathrm{~b}$. Airtraq view of the larynx in a 5-year-old patient. (a) The device is rotated $15^{\circ}$ to the left. (b) Note that both cords are easily seen but the tracheal tube is not aligned with the tracheal axis. Intubation is likely to fail in this position.

in the trachea by DL on second attempt. In general, a thin airway exchange catheter or bougie could be used as an adjunct to DL to probe the glottic opening. This combination could result in a more careful intubation of the pediatric airway compared to the AT intubation.

Our study has several limitations. The first limitation is that the study was not blinded because it is clearly not possible to blind the operator to the device being used. In addition, the anesthesiologists had limited manikin training and only five human exposures to the AT prior to the study, compared to years of experience with DL. One recent study suggested that a minimum of 47 successful exposures to DL are required to achieve basic competence [19]. It has not been determined how many successful exposures are required to achieve basic 


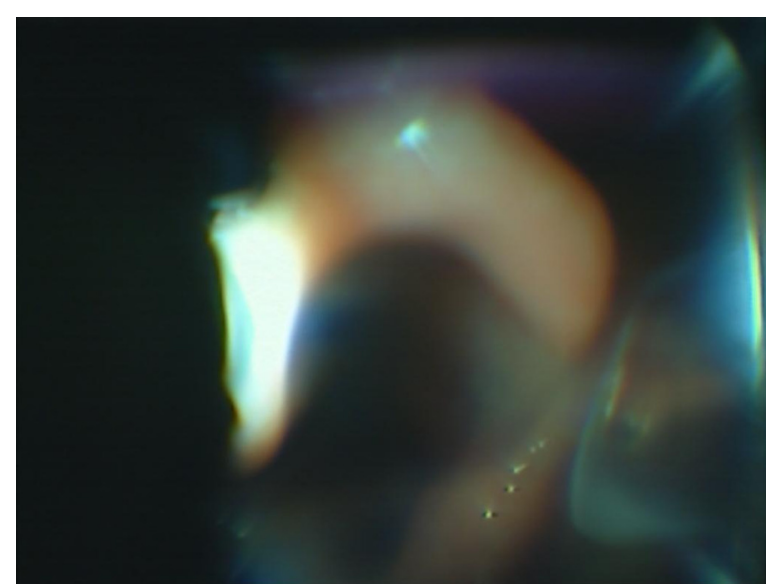

(a)

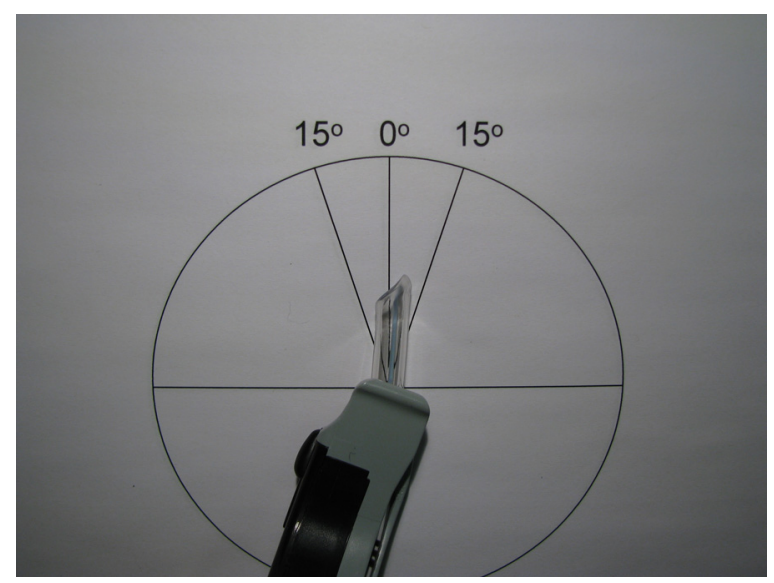

(b)

Figs $3 \mathrm{a}$ and $3 \mathrm{~b}$. Airtraq view of the larynx in same patient as Figure 2a. (a) The device is rotated $15^{\circ}$ to right. (b) Note that the view demonstrates only the right vocal cord but the tracheal tube is aligned with the tracheal axis. Intubation is likely to succeed in this position.

competence with the AT. The inability to blind the operators combined with the relative inexperience of the operators with the AT device clearly introduces a significant performance bias in favor of DL. Given this bias, it is interesting to note that the anesthesiologists obtained better laryngeal views with the new device than with the standard laryngoscope, and achieved best view in a comparable time. It is necessary to note, that in this study the anesthesiologist did not lift the epiglottis with the straight blade, which could have resulted in lower POGO scores in the DL group. The comparison of the laryngoscopic views has to be interpreted with caution. However, the finding of improved view with comparable or prolonged intubation time is similar to what has been described for other indirect laryngoscopy devices [11-18]. It remains to be determined whether further practice and experience with the pediatric AT will allow the better view to translate into faster, more successful intubation. Given that the AT is a single use device, training and acquiring expertise is associated with increased cost. A cost of $\$ 80$ US (year 2007) is expensive for a single use primary airway management device with a considerable learning curve [20]. It is also clear that further research is needed to assess the efficacy of the AT in pediatric patients with difficult airway anatomy. For now, indirect laryngoscopy devices like the AT are thought to be used rather in the unexpected difficult airway. The expected difficult pediatric airway should be managed by fiberoptic tracheal intubation via laryngeal mask as presented by Weiss and Engelhardt [21], to maintain skills in this important technique.

In summary, our observations lead us to conclude that the AT optical laryngoscope can quickly and easily provide superb views of the pediatric larynx. This may prove to be valuable in managing difficult pediatric airways. While our initial experience is encouraging, pediatric AT intubation is not quite as easy as hoped. Learning the basic AT technique and the required pediatric modifications takes practice and experience.

\section{References}

[1] Mamie C, Habre W, Delhumeau C, Argiroffo CB, Morabia A. Incidence and risk factors of perioperative respiratory adverse events in children undergoing elective surgery. Paediatr Anaesth 2004;14:218-24.

[2] Maharaj CH, Higgins BD, Harte BH, Laffey JG. Evaluation of intubation using the Airtraq or Macintosh laryngoscope by anaesthetists in easy and simulated difficult laryngoscopya manikin study. Anaesthesia 2006;61:469-77.

[3] Savoldelli GL, Schiffer E, Abegg C, Baeriswyl V, Clergue F, Waeber JL. Learning curves of the Glidescope, the McGrath and the Airtraq laryngoscopes: a manikin study. Eur J Anaesthesiol 2009;26:554-8.

[4] Maharaj CH, O'Croinin D, Curley G, Harte BH, Laffey JG. A comparison of tracheal intubation using the Airtraq or the Macintosh laryngoscope in routine airway management: A randomised, controlled clinical trial. Anaesthesia 2006;61:1093-9.

[5] Maharaj CH, Costello JF, Harte BH, Laffey JG. Evaluation of the Airtraq and Macintosh laryngoscopes in patients at increased risk for difficult tracheal intubation. Anaesthesia 2008;63:182-8.

[6] Maharaj CH, Buckley E, Harte BH, Laffey JG. Endotracheal intubation in patients with cervical spine immobilization: a comparison of macintosh and airtraq laryngoscopes. Anesthesiology 2007;107:53-9. 
[7] Turkstra TP, Pelz DM, Jones PM. Cervical spine motion: a fluoroscopic comparison of the AirTraq Laryngoscope versus the Macintosh laryngoscope. Anesthesiology 2009;111:97-101.

[8] Dhonneur G, Ndoko S, Amathieu R, Housseini LE, Poncelet C, Tual L. Tracheal intubation using the Airtraq in morbid obese patients undergoing emergency cesarean delivery. Anesthesiology 2007;106:629-30.

[9] Malin E, Montblanc J, Ynineb Y, Marret E, Bonnet F. Performance of the Airtraq laryngoscope after failed conventional tracheal intubation: a case series. Acta Anaesthesiol Scand 2009;53:858-63.

[10] Hirabayashi Y, Shimada N, Nagashima S. Tracheal intubation using pediatric Airtraq optical laryngoscope in a patient with Treacher Collins syndrome. Paediatr Anaesth 2009; 19:915-6.

[11] Péan D, Desdoits A, Asehnoune K, Lejus C. Airtraq laryngoscope for intubation in Treacher Collins syndrome. Paediatr Anaesth 2009;19:698-9.

[12] Weiss M, Schwarz U, Dillier CM, Gerber AC. Teaching and supervising tracheal intubation in paediatric patients using videolaryngoscopy. Paediatr Anaesth 2001;11:343-8.

[13] Macnair D, Baraclough D, Wilson G, Bloch M, Engelhardt T. Pediatric airway management: comparing the BerciKaplan Video Laryngoscope with direct laryngoscopy. Paediatr Anaesth 2009;19:577.
[14] Vlatten A, Aucoin S, Litz S, Macmanus B, Soder C. A comparison of the STORZ video laryngoscope and standard direct laryngoscopy for intubation in the Pediatric airway-a randomized clinical trial. Paediatr Anaesth 2009;19:1102-7.

[15] Kim JT, Na HS, Bae JY, Kim DW, Kim HS, Kim CS, et al. GlideScope video laryngoscope: a randomized clinical trial in 203 paediatric patients. Br J Anaesth 2008;101:531-4.

[16] Singh R, Singh P, Vajifdar H. A comparison of Truview infant EVO2 laryngoscope with the Miller blade in neonates and infants. Paediatr Anaesth 2009;19:338-42.

[17] Dhonneur G, Abdi W, Amathieu R, Ndoko S, Tual L. Optimising tracheal intubation success rate using the Airtraq laryngoscope. Anaesthesia 2009;64:315-9.

[18] Xue FS, He N, Liu JH, Liao X, Xu XZ, Zhang YM. More maneuvers to facilitate endotracheal intubation using the Airtraq laryngoscope in children with difficult airways. Paediatr Anaesth 2009;19:916-8.

[19] Mulcaster JT, Mills J, Hung OR, MacQuarrie K, Law JA, Pytka S, et al. Laryngoscopic intubation: learning and performance. Anesthesiology 2003;98:23-7.

[20] Dhonneur G, Ndoko Sk. Comparing the Airtraq ${ }^{\circledR}$ with the LMA CTrach ${ }^{\mathrm{TM}}$. Anesthesiology 2007;107:675-6.

[21] Weiss M, Engelhardt T. Proposal for the management of the unexpected difficult pediatric airway. Paediatr Anaesth 2010;20:454-64. 